\title{
Do brand characteristics cont ribute significantly to variability in toxicant exposure in smokers? Data from NHANES 2007-2012
}

\author{
Richard J O'Connor', Liane M Schneller', Lynn T Kozlowski
}

\begin{abstract}
INTRODUCTION This study sought to quantify the difference in serum cotinine and other biomarkers indicative of cigarette smoking in current US cigarette smokers attributable to brand level versus individual level factors.

METHODS A total of 2,558 daily exclusive smokers, 20 years and older in the United States participated in the National Health and Nutrition Examination Surveys 2007-2012 and provided biospecimens and cigarette brand information. Biomakers of interest were serum cotinine, and urinary NNAL, PAHs, and heavy metals. Adjustments were made for person-level factors (e.g., sex, age, race, education), smoking behavior (cigarettes per day), brand, tar group ( $\leq 6 \mathrm{mg}$, $>6-15 \mathrm{mg},>15 \mathrm{mg})$, and menthol status.

RESULTS The most commonly reported brands of US cigarettes were Marlboro, Newport, Camel, and Pall Mall. Cotinine levels differed by age $(p=0.0065)$, race $(p<0.0001)$, and cigarettes smoked per day $(\mathrm{p}<0.0001)$ but not brand, tar or menthol. Brand family was significantly associated with urinary levels of NNAL, 1HP, HFs, and HPHs. For NNAL. Person-level factors accounted for some of these differences. No consistent differences in metal exposure by brand were noted. Overall, brand information accounted for $2-8 \%$ of variance depending on the marker. Together, age, sex, race, education, and cigarettes smoked per day accounted for $20 \%$ in variance in cotinine levels, and adding the brand information (brand family, tar group, menthol) to the model accounted for an additional $0.5 \%$ ( $\mathrm{p}<0.0948)$. In the full multivariate model, no significant difference in cotinine was seen by brand $(\mathrm{p}=0.2205)$, $\operatorname{tar}(\mathrm{p}=0.2987)$ or menthol $(\mathrm{p}=0.1583)$. Conclusions Brand to brand differences seen in serum cotinine levels and other biomarkers indicative of cigarette smoking between the top US cigarette brands from 2007-2012 are primarily driven by individual differences among smokers, and to a lesser extent by differences among products.
\end{abstract}

\section{INTRODUCTION}

Cigarette smoking remains a leading cause of death and disability, with smoke containing thousands of compounds ${ }^{1-3}$, including addictive nicotine ${ }^{4-6}$. In response to reductions in cigarette nicotine availability, smokers will compensate by numbers of cigarettes smoked or increased puffing and inhalation $^{6}$, maintaining or even increasing their exposure to toxicants. Assessing smoke exposure in humans is commonly achieved by using biomarkers, most commonly metabolites of smoke constituents, such as cotinine (nicotine) $)^{7-10}$, 4-(methylnitrosamino)-1-(3-pyridyl)-1-butanol (NNAL) for the tobacco-specific n-nitrosamine 4-(methylnitrosamino)- 1-(3-pyridyl)-1-butanone (NNK $)^{11-13}$, and the hydroxylated derivatives of a number of polycyclic aromatic hydrocarbons (PAH), such as pyrene, naphthalene, and phenanthrene ${ }^{14}$. Tobacco is also a point source for heavy metal exposures ${ }^{15-17}$, including $\mathrm{Pb}$, As, and $\mathrm{Cd}$. Cadmium in particular is consistently shown to be elevated among smokers ${ }^{18-20}$.

Few studies have looked at the difference in brand-specific exposures from cigarettes. The existing literature looks at broader categories (e.g., Light vs Full Flavor), and generally finds no substantial difference in exposure between products of different standard tar yields, for example ${ }^{21,22}$. However, consumers tend to select a brand of cigarettes and to stick 
with that brand ${ }^{23}$. Marlboro holds the highest market share for leading cigarette brands in the United States (US) followed by Newport, Camel and Pall Mall, together accounting for over $60 \%$ of the market ${ }^{24}, 25$. Tobacco industry marketing tactics, including advertising, packaging, and design/engineering influence brand preference ${ }^{21-26}$. Marlboro, Newport and Camel are the three most advertised cigarettes brands and the preferred brands among young cigarette smokers ${ }^{21-26}$. Having data at a brand specific level is potentially relevant as tobacco products are now under regulation by the Food and Drug Administration (FDA). Manufacturers must test for and report on a variety of harmful and potentially harmful constituents (HPHC), and must submit reports to justify any changes to an existing product or the introduction of a new product (substantial equivalence) ${ }^{27}$. HPHCs include NNK, PAHs, and metals ${ }^{28}$. FDA also has authority to set performance standards for products, including on HPHC content or emission ${ }^{27}$. So establishing a 'typical' exposure among users of very popular products is important for informing such regulations moving forward. At the same time, the existing literature suggest that there is little difference in smokers' exposures to toxic constituents by nominal tar yield or type, so quantifying the degree to which brand differences (such as brand family, nominal tar yield, or menthol) account for inter-individual variability in specific HPHCs might provide a focal point for regulation.

The National Health and Nutrition Examination Survey (NHANES) was designed to monitor the health, nutrition and health behavior of adults and children in the United States $^{29,30}$. The NHANES provides selected disease and risk factor prevalence data and monitors disease, behavior and environmental exposure trends in the US, including serum cotinine ${ }^{29,30}$. This study aims to quantify the difference in serum cotinine levels and other biomarkers indicative of cigarette smoke exposure in current US cigarette smokers according to the top US cigarette brands using NHANES 2007-2012 data.

\section{METHODS}

\section{Survey design and study population}

Composed of interviews and physical examinations, the NHANES program is a part of the National Center for Health Statistics (NCHS) under the Centers for Disease Control and Prevention $(\mathrm{CDC})^{29,30}$. Its sample population (about 5,000 respondents per year) aims to represent all ages of the noninstitutionalized US population, and in order to produce reliable statistics, persons 60 and older, African Americans and Hispanics, and low income White Americans are over- sampled $^{29-31}$. Sample weights are assigned to each participant to allow unbiased, national estimates to be derived ${ }^{32}$. The personal health interviews are performed in the respondent's home, and the standardized health evaluation at a mobile examination center (MEC) several days later ${ }^{29}$. The NCHS Research Ethics Review Board (ERB) approved the survey protocol and participants provided consent ${ }^{32}$.

Smoking history and characteristics were collected from adults, 20 years and older, during the household interview ${ }^{33}$. Preferred brand of cigarettes was determined at the MEC visit and confirmed using the uniform product codes (UPC) ${ }^{33}$. For this study, smokers were categorized according to their preferred brand among the top US filtered cigarette brands (Marlboro, Newport, Camel, Pall Mall, Other); non-US brands and unfiltered cigarettes were excluded from the analysis. From this information, the manufacturer and price category was determined ${ }^{34}$. Demographic characteristics including age, gender, race, education, marital status, and income were also collected during the interview. This study sample was limited to participants who reported daily use of cigarettes but no other tobacco product or nicotine replacement therapy (NRT) and provided blood for serum cotinine measurement during the 2007, 2009 or 2011 NHANES. There were 2,558 such smokers in NHANES 2007-2012 who were 20 years or older (2007: 36.5\%; 2009: 33.9\%; 2011: 29.6\%). They were primarily married men that were Non-Hispanic White, with some college or an Associate's degree, and living above the poverty line (See Table 1).

\section{Laboratory Analysis}

Blood and urine collection was done at the MEC visit; details on sample collection, processing, quality assurance and quality control instructions can be found elsewhere ${ }^{30-38}$. Cotinine was measured in the serum while NNAL and PAHs (2-, 3-, and 9-hydroxyfluorene, 1-, 2-, and 3-hydroxyphenanthrene, 1-hydroxypyrene, and 2-hydroxynaphthalene ) were measured in the urine using an isotope dilution-high performance liquid chromatography / atmospheric pressure chemical ionization tandem mass spectrometry (ID HPLC-APCI MS/MS) ${ }^{36}$. Urinary metal (arsenic, As; cadmium, Cd; cobalt, Co; lead, $\mathrm{Pb}$; and mercury, $\mathrm{Hg}$ ) concentrations were determined using inductively coupled plasma-mass spectrometry (ICP-MS) ${ }^{37}$. When results were below the limit of detection, the detection limit divided by the square root of two was imputed ${ }^{36,37}$. Results from urine assays were creatinine $(\mathrm{Cr})$ adjusted and presented in units/mg of creatinine. Also during the participants MEC visit, body measurements for height and weight were collected and used to calculate BMI. 


\section{Statistical Analysis}

Statistical analyses were conducted using NHANES weights to approximate the US population with SAS 9.4 (SAS Institute, Inc., Cary, North Carolina) and SUDAAN 11.0.1 (RTI International, Research Triangle Park, North Carolina) software to account for the complex sampling design. Multiyear weights were calculated as recommended. For cotinine and NNAL, which were assayed for the whole sample, the MEC weights were used. For the PAH and metal analyses, assays were performed only on a randomly selected subsample, and so corresponding weights were used. Proportions are presented for categorical variables and averages and standard errors for continuous variables according to preferred cigarette brands. Multivariate linear regression was used to examine serum cotinine $(\mathrm{N}=2,558)$, urine NNAL $(\mathrm{N}=2,412)$, urine 2-hydroxynaphthalene ( $2 \mathrm{HN} ; \mathrm{N}=220$ ), urine 1-hydroxypyrene (1HP; N=220), total urine hydroxyfluorenes (Sum HF; $\mathrm{N}=220$ ), total urine hydroxyphenanthrenes (Sum HPH; $\mathrm{N}=220)$, urine As ( $\mathrm{N}=833)$, $\mathrm{Cd}(\mathrm{N}=837)$, Co $(\mathrm{N}=837), \mathrm{Pb}$ ( $\mathrm{N}=837)$, and $\mathrm{Hg}(\mathrm{N}=838)$. Federal Trade Commission (FTC) tar was categorized into three groups (Less than or equal to
$6 \mathrm{mg}, 6.1$ to $15 \mathrm{mg}$, and greater than $15 \mathrm{mg}$ ) corresponding to historical cutpoints of 'ultra-light,' 'light,' and 'full flavor' varieties, as these may retain consumer salience even if use of the descriptors have been restricted. Models were adjusted for gender, age, race/ethnicity, education, and cigarettes per day (CPD). Additional models controlled for brand family, tar levels, and menthol status. Cotinine was also adjusted for in all models, except when cotinine was the dependent variable. Marlboro brand cigarettes were used as the referent group. R-squared for models containing brand variables (brand family, tar group, menthol) were compared to models with these variables omitted to estimate the proportion of variance explained by these factors. The difference in R-square between these models was evaluated against the $\mathrm{F}$ distribution.

\section{RESULTS}

Between 2007 and 2012, the most commonly reported brand of US cigarettes among daily smokers was Marlboro (37.6\%) followed by Newport (14.5\%), Camel (6.6\%), and then Pall Mall (5.8\%). There was some variability across the study span. Marlboro dropped from $39.2 \%$ in 2007 to $35.0 \%$ in

\section{Table 1: Weighted Demographics of the NHA NES 2007-2012 Cigarette Smokers According to Preferred Cigarette Brand}

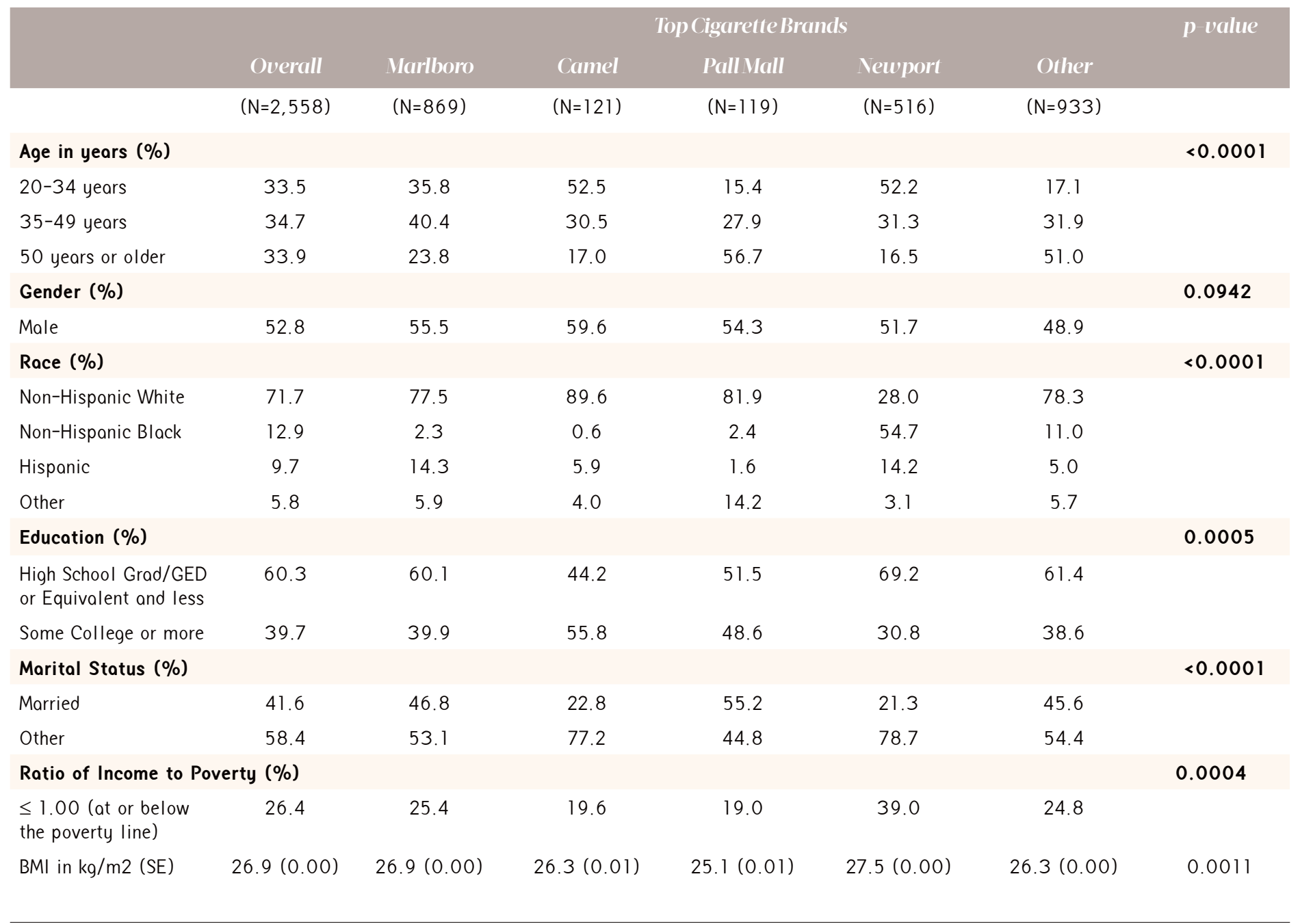




\section{Cigarettes per Day (\%)}

5 cigarettes or less

6-15 cigarettes

16 cigarettes or more

Serum Cotinine in ng/ $\mathrm{mL}$ (SE)

Tar Level (\%)

$\leq 6 \mathrm{mg}$
$>6-15 \mathrm{mg}$

$>15 \mathrm{mg}$

Menthol Status (\%)

Mentholated

\section{4}

39.1

41.5

199.5

$(0.01)$

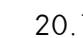

$$
40.3
$$

$177.8(0.02)$

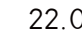

43.1

34.9

$182.0(0.04)$
8.4

36.1

55.6

$245.5(0.02)$

$<0.0001$

$15.8 \quad 19$.

$60.9 \quad 73.7$

7.3

\section{2}

44.4

47.4

15.4

17.3

0.5

27.6

71.9

75.6
14.6

33.7

51.8

$218.8(0.02)$

$<0.0001$

$<0.0001$

$<0.0001$

Table 2. Regression weights for demographics, CPI, and cotinine as predictors for exposure biomarkers, NHANES 2007-12.

\begin{tabular}{|c|c|c|c|c|c|c|c|c|c|c|}
\hline & NNIL & IIIP & $2 / \mathrm{N}$ & Sum IIF & Sum IIPII & As & Cd & Co & $\mathbf{P b}$ & $\mathrm{Ig}$ \\
\hline $\begin{array}{l}\text { Age in } \\
\text { years }\end{array}$ & $p<0.0001$ & $p=0.3615$ & $p=0.7376$ & $p=0.0031$ & $p=0.4145$ & $p=0.0002$ & $p<0.0001$ & $p=0.0012$ & $p<0.0001$ & $p=0.0010$ \\
\hline $\begin{array}{l}20-34 \\
\text { years }\end{array}$ & REF & REF & REF & REF & REF & REF & REF & REF & REF & REF \\
\hline $\begin{array}{l}35-49 \\
\text { years }\end{array}$ & 0.16 & 0.07 & 0.07 & 0.12 & 0.13 & 0.14 & 0.29 & 0.05 & 0.16 & 0.10 \\
\hline $\begin{array}{l}50 \text { years } \\
\text { or older }\end{array}$ & 0.20 & 0.04 & 0.06 & 0.13 & 0.13 & 0.14 & 0.44 & 0.09 & 0.23 & 0.12 \\
\hline Gender & $p<0.0001$ & $p<0.0001$ & $p<0.0001$ & $p<0.0001$ & $p=0.0045$ & $p=0.0708$ & $p<0.0001$ & $p<0.0001$ & $p=0.1130$ & $p=0.0013$ \\
\hline Male & 0.16 & 0.13 & 0.15 & 0.11 & 0.08 & -0.08 & 0.12 & 0.14 & -0.04 & 0.08 \\
\hline Race & $p<0.0001$ & $p=0.0006$ & $p=0.0011$ & $p=0.0003$ & $p=0.0016$ & $p<0.0001$ & $p=0.2196$ & $p<0.0001$ & $p=0.0476$ & $p=0.0092$ \\
\hline $\begin{array}{l}\text { Non- } \\
\text { Hispanic } \\
\text { White }\end{array}$ & REF & REF & REF & REF & REF & REF & REF & REF & REF & REF \\
\hline $\begin{array}{l}\text { Non- } \\
\text { Hispanic } \\
\text { Black }\end{array}$ & -0.23 & -0.31 & -0.16 & -0.23 & -0.27 & 0.16 & 0.00 & -0.14 & -0.05 & -0.11 \\
\hline Hispanic & -0.07 & -0.03 & 0.06 & 0.00 & 0.00 & 0.14 & 0.02 & -0.06 & 0.05 & -0.06 \\
\hline Other & -0.10 & 0.02 & 0.00 & -0.01 & -0.02 & 0.40 & 0.08 & 0.01 & 0.08 & 0.04 \\
\hline Education & $p=0.0001$ & $p=0.8015$ & $p=0.1581$ & $p=0.5608$ & $p=0.6761$ & $p=0.1535$ & $p=0.0075$ & $p=0.9800$ & $p=0.1363$ & $p=0.0003$ \\
\hline $\begin{array}{l}\text { High } \\
\text { School } \\
\text { Grad/ } \\
\text { GED or } \\
\text { Equivalent } \\
\text { and less }\end{array}$ & 0.06 & 0.01 & 0.01 & 0.02 & -0.07 & -0.06 & 0.07 & 0.00 & 0.03 & -0.08 \\
\hline $\begin{array}{l}\text { Cigarettes } \\
\text { per Day }\end{array}$ & $p<0.0001$ & $p=0.3736$ & $p=0.0565$ & $p=0.2468$ & $p=0.4301$ & $p=0.5192$ & $p=0.0565$ & $p=0.5947$ & $p=0.4324$ & $p=0.2300$ \\
\hline $\begin{array}{l}5 \\
\text { cigarettes } \\
\text { or less }\end{array}$ & REF & REF & REF & REF & REF & REF & REF & REF & REF & REF \\
\hline $\begin{array}{l}6-15 \\
\text { cigarettes }\end{array}$ & 0.09 & 0.03 & 0.00 & 0.02 & 0.01 & -0.03 & 0.04 & -0.01 & -0.02 & 0.00 \\
\hline $\begin{array}{l}16 \\
\text { cigarettes } \\
\text { or more }\end{array}$ & 0.21 & 0.08 & 0.07 & 0.07 & 0.00 & 0.01 & 0.08 & -0.02 & 0.01 & -0.05 \\
\hline $\begin{array}{l}\text { Serum } \\
\text { cotinine }\end{array}$ & $p<0.0001$ & $p<0.0001$ & $p<0.0001$ & $p<0.0001$ & $p<0.0001$ & $p=0.0581$ & $p=0.0044$ & $p=0.2155$ & $p=0.0430$ & $p=0.0774$ \\
\hline Continuous & 0.52 & 0.46 & 0.48 & 0.60 & 0.42 & -0.09 & 0.09 & 0.03 & 0.06 & -0.08 \\
\hline
\end{tabular}

$P$ value represents Wald $F$ test; Bolded regression coefficients are significant at $p<0.05$ by $t$-test 
Table 3. Model R-Squarechange

\begin{tabular}{|c|c|c|c|c|c|c|c|c|}
\hline & $\mathbf{N}$ & $\begin{array}{c}\text { R Square Value } \\
\text { Demographics Only }\end{array}$ & Add CPD & $p$ & Idd Cotinine & $p$ & Add Brand Features & $p$ \\
\hline Cotinine & 2558 & 0.07 & 0.20 & $<.001$ & $x x x$ & $x x x$ & 0.20 & 0.052 \\
\hline NNAL & 2412 & 0.19 & 0.30 & $<.001$ & 0.48 & $<.001$ & 0.50 & $<.001$ \\
\hline $1-H P$ & 220 & 0.17 & 0.23 & 0.001 & 0.35 & $<.001$ & 0.43 & $<.001$ \\
\hline $2-\mathrm{HN}$ & 220 & 0.15 & 0.25 & $<.001$ & 0.45 & $<.001$ & 0.50 & 0.007 \\
\hline sum HF & 220 & 0.19 & 0.29 & $<.001$ & 0.54 & $<.001$ & 0.59 & 0.007 \\
\hline sum $\mathrm{HPH}$ & 220 & 0.20 & 0.23 & 0.030 & 0.39 & $<.001$ & 0.46 & 0.001 \\
\hline As & 833 & 0.10 & 0.10 & 0.407 & 0.10 & 0.028 & 0.12 & 0.330 \\
\hline $\mathrm{Cd}$ & 837 & 0.46 & 0.48 & $<.001$ & 0.49 & $<.001$ & 0.51 & $<.001$ \\
\hline Co & 837 & 0.17 & 0.17 & 0.853 & 0.17 & 0.113 & 0.18 & 0.703 \\
\hline $\mathrm{Pb}$ & 837 & 0.15 & 0.15 & 0.134 & 0.16 & 0.021 & 0.17 & 0.725 \\
\hline $\mathrm{H} 9$ & 838 & 0.09 & 0.10 & 0.003 & 0.11 & 0.009 & 0.13 & 0.029 \\
\hline
\end{tabular}

Note: P-Values from F-test comparing difference in R-square from consecutive models. CPD = cigarettes smoked per day.

Table 4. Mean creatinine adjusted concentrations of various HPIC biomarkers in current smokers by brand, tar group, and menthol status, NHA NES 2007-2012.

\begin{tabular}{|c|c|c|c|c|c|c|c|c|c|c|c|c|c|c|c|}
\hline \multirow[b]{3}{*}{ Brand } & \multicolumn{3}{|c|}{ NNAL (ng mg) } & \multicolumn{3}{|c|}{ IIIP (ng mg) } & \multicolumn{3}{|c|}{ 2IIP (ng mg) } & \multicolumn{3}{|c|}{ Sum IIF (ng mg) } & \multicolumn{3}{|c|}{ Sum IIPI (ng mg) } \\
\hline & G. Mean & $\mathrm{LCL}$ & UCL & G. Mean & $\mathrm{LCL}$ & UCL & G. Mean & $\mathrm{LCL}$ & UCL & G. Mean & LCL & UCL & G. Mean & $\mathrm{LCL}$ & UCL \\
\hline & \multicolumn{3}{|c|}{$p<0.0001$} & \multicolumn{2}{|c|}{$p=0.0073$} & & \multicolumn{3}{|c|}{$p=0.0609$} & \multicolumn{3}{|c|}{$p=0.0310$} & \multicolumn{3}{|c|}{$p=0.0227$} \\
\hline Marlboro & 0.34 & 0.32 & 0.36 & 0.31 & 0.26 & 0.37 & 14.13 & 12.88 & 15.85 & 3.02 & 2.69 & 3.47 & 0.58 & 0.50 & 0.66 \\
\hline Camel & 0.23 & 0.20 & 0.28 & 0.32 & 0.22 & 0.48 & 13.18 & 9.12 & 19.05 & 2.69 & 1.91 & 3.80 & 0.51 & 0.36 & 0.72 \\
\hline Pall Mall & 0.31 & 0.27 & 0.35 & 0.58 & 0.37 & 0.87 & 18.20 & 15.14 & 21.88 & 4.57 & 3.80 & 5.50 & 0.91 & 0.72 & 1.15 \\
\hline Newport & 0.23 & 0.21 & 0.26 & 0.39 & 0.30 & 0.50 & 17.78 & 13.80 & 23.44 & 3.72 & 2.95 & 4.57 & 0.63 & 0.49 & 0.81 \\
\hline Other & 0.30 & 0.26 & 0.33 & 0.28 & 0.25 & 0.31 & 17.38 & 15.85 & 19.50 & 3.39 & 3.02 & 3.80 & 0.58 & 0.52 & 0.63 \\
\hline Tar & \multicolumn{3}{|c|}{$p=0.4798$} & \multicolumn{2}{|l|}{$p=0.0071$} & & \multicolumn{2}{|c|}{$p=0.0451$} & & \multicolumn{3}{|c|}{$p=0.0996$} & \multicolumn{3}{|c|}{$p=0.0201$} \\
\hline$\leq 6 \mathrm{mg}$ & 0.31 & 0.27 & 0.36 & 0.36 & 0.32 & 0.43 & 17.38 & 14.79 & 20.42 & 3.80 & 3.31 & 4.47 & 0.72 & 0.62 & 0.85 \\
\hline $6.1-15 \mathrm{mg}$ & 0.29 & 0.27 & 0.32 & 0.35 & 0.30 & 0.42 & 16.60 & 15.14 & 18.20 & 3.24 & 2.88 & 3.63 & 0.60 & 0.52 & 0.69 \\
\hline$>15 \mathrm{mg}$ & 0.30 & 0.28 & 0.34 & 0.26 & 0.23 & 0.31 & 13.80 & 11.75 & 16.22 & 3.09 & 2.57 & 3.63 & 0.54 & 0.46 & 0.62 \\
\hline Menthol & \multicolumn{3}{|c|}{$p=0.0412$} & \multicolumn{2}{|l|}{$p=0.0877$} & & \multicolumn{2}{|c|}{$p=0.7297$} & & \multicolumn{3}{|c|}{$p=0.9638$} & \multicolumn{3}{|c|}{$p=0.7910$} \\
\hline No & 0.31 & 0.28 & 0.33 & 0.30 & 0.27 & 0.34 & 15.85 & 14.45 & 17.38 & 3.31 & 2.95 & 3.63 & 0.59 & 0.54 & 0.66 \\
\hline \multirow[t]{3}{*}{ Yes } & 0.28 & 0.25 & 0.31 & 0.39 & 0.30 & 0.52 & 15.49 & 12.30 & 19.05 & 3.24 & 2.57 & 4.07 & 0.62 & 0.49 & 0.76 \\
\hline & \multicolumn{3}{|c|}{ As (pg/mg) } & \multicolumn{3}{|c|}{$\mathrm{Cd}(\mathrm{pg} / \mathrm{mg})$} & \multicolumn{3}{|c|}{ Co (pg/mg) } & \multicolumn{3}{|c|}{$\mathrm{Pb}(\mathrm{pg} / \mathrm{mg})$} & & $(\mathrm{pg} / \mathrm{m}$ & \\
\hline & G. Mean & $\mathrm{LCL}$ & UCL & G. Mean & $\mathrm{LCL}$ & UCL & G. Mean & $\mathrm{LCL}$ & UCL & G. Mean & $\mathrm{LCL}$ & UCL & G. Mean & $\mathrm{LCL}$ & UCL \\
\hline Brand & $p=0.584$ & & & $p=0.6117$ & & & $p=0.817$ & & & $p=0.614$ & & & $p=0.40$ & & \\
\hline Marlboro & 7.94 & 6.76 & 9.33 & 0.50 & 0.46 & 0.54 & 0.48 & 0.45 & 0.51 & 0.72 & 0.65 & 0.81 & 0.51 & 0.46 & 0.59 \\
\hline Camel & 6.46 & 5.01 & 8.13 & 0.52 & 0.35 & 0.78 & 0.50 & 0.40 & 0.65 & 0.69 & 0.56 & 0.83 & 0.56 & 0.44 & 0.72 \\
\hline Pall Mall & 7.41 & 5.13 & 10.47 & 0.51 & 0.43 & 0.62 & 0.49 & 0.40 & 0.60 & 0.83 & 0.69 & 1.02 & 0.41 & 0.32 & 0.52 \\
\hline Newport & 8.71 & 7.08 & 10.96 & 0.50 & 0.44 & 0.58 & 0.50 & 0.44 & 0.56 & 0.72 & 0.56 & 0.93 & 0.54 & 0.45 & 0.63 \\
\hline Other & 7.94 & 6.76 & 9.12 & 0.55 & 0.50 & 0.59 & 0.50 & 0.47 & 0.55 & 0.78 & 0.71 & 0.83 & 0.51 & 0.46 & 0.59 \\
\hline Tar & $p<0.02 c$ & & & $p<0.3983$ & & & $p<0.653$ & & & $p<0.490$ & & & $p<0.063$ & & \\
\hline$\leq 6 \mathrm{mg}$ & 9.33 & 7.94 & 10.96 & 0.56 & 0.48 & 0.65 & 0.51 & 0.46 & 0.56 & 0.71 & 0.63 & 0.78 & 0.60 & 0.51 & 0.69 \\
\hline $6.1-15 \mathrm{mg}$ & 7.41 & 6.76 & 8.13 & 0.51 & 0.49 & 0.55 & 0.49 & 0.47 & 0.52 & 0.74 & 0.69 & 0.79 & 0.49 & 0.45 & 0.54 \\
\hline$>15 \mathrm{mg}$ & 8.32 & 6.76 & 10.00 & 0.49 & 0.44 & 0.56 & 0.48 & 0.42 & 0.54 & 0.79 & 0.68 & 0.91 & 0.52 & 0.43 & 0.65 \\
\hline Menthol & $p=0.249$ & & & $p=0.0045$ & & & $p=0.268$ & & & $p=0.919$ & & & $\mathrm{p}=0.348$ & & \\
\hline No & 8.13 & 7.24 & 9.33 & 0.55 & 0.51 & 0.58 & 0.50 & 0.47 & 0.54 & 0.74 & 0.71 & 0.79 & 0.52 & 0.48 & 0.58 \\
\hline Yes & 7.24 & 6.17 & 8.51 & 0.46 & 0.42 & 0.51 & 0.47 & 0.43 & 0.51 & 0.74 & 0.65 & 0.87 & 0.49 & 0.42 & 0.56 \\
\hline
\end{tabular}

NOTE: All mean values adjusted for participant sex, age, race, education, cigarettes smoked per day, and serum cotinine 
2011; Newport rose from $12.5 \%$ to $14.2 \%$ over $2007-2011$. Camel (6.2\% in 2007 vs. $8.6 \%$ in 2011$)$ and Pall Mall (2.9\% in 2007 vs $9.6 \%$ in 2011) also rose in popularity. These changes were found to be statistically significant $(=2.26, \mathrm{p}=0.00387$ ) and are consistent with prior reports ${ }^{24}$. Current smokers who preferred Marlboro cigarettes tended to be Non-Hispanic White males who were 35-49 years of age, have graduated high school/received a GED or less, and smoke about 6-15 cigarettes per day. Camel cigarettes were most popular among Non-Hispanic White men who were 20-34 years of age, had some college or an Associate's degree, and smoke about 6-15 cigarettes per day. Participants who preferred Pall Mall were primarily Non-Hispanic White males who were 50 years of age or older, have graduated high school/received a GED or less, and smoke 16 cigarettes or more per day. Last, current US smokers who preferred Newport cigarettes were Non-Hispanic Black males, 20-34 years of age, have graduated high school/ received a GED or less, and smoke about 6-15 cigarettes per day (See Table 1). Tar levels and menthol status were found to be differentially distributed among these leading US brands $(\mathrm{p}<0.0001)$.

Geometric mean serum cotinine across the sample was $199.5 \mathrm{ng} / \mathrm{mL} 1$. There were no significant changes observed in mean serum cotinine levels across the three survey periods. Differences were seen among participants who smoked Pall Mall, Newport and other US cigarette brands in relation to those who preferred Marlboro cigarettes (Marlboro $=177.8 \mathrm{ng}$ / $\mathrm{mL}$; Pall Mall $=245.5 \mathrm{ng} / \mathrm{mL}, \mathrm{p}<0.0001$; Newport $=204.2 \mathrm{ng} /$ $\mathrm{mL}, \mathrm{p}=0.0160$; Other $=218.8 \mathrm{ng} / \mathrm{mL}, \mathrm{p}=0.0005$ ). Table 1 displays the unadjusted geometric mean serum cotinine levels by preferred brand of cigarettes. The highest cotinine levels were found among smokers who preferred Pall Mall, followed by Newport, Camel and Marlboro. Cotinine levels differed significantly from Marlboro brand cigarettes for Pall Mall $(p<0.0001)$, Newport $(p<0.0001)$, and the other brands of US cigarettes $(p<0.0001)$, see Table 1. In multivariate models, cotinine levels differed significantly by age $(p=0.0065)$, race $(p<0.0001)$, and cigarettes smoked per day $(p<0.0001)$, but not gender $(\mathrm{p}=0.8727)$ or education $(\mathrm{p}=0.1048)$. Relative to smokers aged 20-34, smokers aged $35-49[\mathrm{~B}=0.05$, SE $=0.02 ; \mathrm{p}=0.0166]$ and $50+[\mathrm{B}=0.07, \mathrm{SE}=0.03 ; \mathrm{p}=0.0083]$ had higher cotinine levels. Compared to smokers of 5 or fewer cigarettes per day, smokers of $6-15$ cigarettes $[B=0.27$, $\mathrm{SE}=0.03, \mathrm{p}<0.0001]$ or 16 or more cigarettes per day $[\mathrm{B}=0.41$, $\mathrm{SE}=0.03 ; \mathrm{p}<0.0001]$ had higher cotinine levels. Relative to white smokers, black smokers $[\mathrm{B}=0.15, \mathrm{SE}=0.02, \mathrm{p}<0.0001]$ had higher cotinine levels, while Hispanic $[\mathrm{B}=-0.15, \mathrm{SE}=0.04$, $\mathrm{p}=0.0004]$ smokers had lower cotinine levels. Together, age, sex, race, education, and cigarettes smoked per day accounted for $20 \%$ in variance in cotinine levels, and adding the brand information (brand family, tar group, menthol) to the model accounted for an additional $0.5 \%(p<0.0948)$. In the full multivariate model, no significant difference in cotinine was seen by brand $(\mathrm{p}=0.2205)$, tar $(\mathrm{p}=0.2987)$ or menthol $(\mathrm{p}=0.1583)$.

NNAL levels differed from Marlboro cigarettes (0.35ng/ $\mathrm{mg} \mathrm{Cr})$ among those who preferred Camel $(0.22 \mathrm{ng} / \mathrm{mg} \mathrm{Cr}, \mathrm{p}$ $<0.0001)$ and Newport $(0.17 \mathrm{ng} / \mathrm{mg}$ Cr, $\mathrm{p}<0.0001)$ brands. 1HP, $2 \mathrm{HN}$ and Sum HF levels differed between Marlboro and Pall Mall brands (1HP: Marlboro $=0.32 \mathrm{ng} / \mathrm{mg}$ Cr, Pall Mall $=0.54 \mathrm{ng} / \mathrm{mg} \mathrm{Cr}, \mathrm{p}=0.0068 ; 2 \mathrm{HN}:$ Marlboro $=15.5 \mathrm{ng} / \mathrm{mg}$ Cr, Pall Mall 18.6ng/mg Cr, p=0.0023; Sum HF: Marlboro = $3.2 \mathrm{ng} / \mathrm{mg}$ Cr, Pall Mall $=4.7 \mathrm{ng} / \mathrm{mg} \mathrm{Cr}, \mathrm{p}<0.0001)$. Those who prefer Pall Mall and Newport brand cigarettes differed by levels of the Sum of HPH compared to that of Marlboro brands (Marlboro $=0.62 \mathrm{ng} / \mathrm{mg}$ Cr; Pall Mall $=0.87 \mathrm{ng} / \mathrm{mg}$ Cr, $\mathrm{p}=0.0050 ;$ Newport $=0.45 \mathrm{ng} / \mathrm{mg}$ Cr, $\mathrm{p}=0.0111)$. Cd levels differed from Marlboro smokers among those who preferred Pall Mall, Newport and other US brands (Marlboro $=0.49 \mathrm{ng} / \mathrm{mg}$ Cr; Pall Mall = 0.60ng $/ \mathrm{mg}$ Cr, p = 0.0339; Newport $=0.38 \mathrm{ng} / \mathrm{mg}$ Cr, p $=0.0039 ;$ Other $=0.62 \mathrm{ng} /$ $\mathrm{mg}$ Cr, $\mathrm{p}=0.0001$ ). Co levels were found to be different among participants who preferred Newport brand cigarettes compared to Marlboro brand smokers (Marlboro $=0.48 \mathrm{ng} / \mathrm{mg}$ $\mathrm{Cr}$, Newport $=0.40 \mathrm{ng} / \mathrm{mg} \mathrm{Cr}, \mathrm{p}=0.0033)$. Pb levels differed from Marlboro brands among Pall Mall cigarettes (Marlboro $=0.71 \mathrm{ng} / \mathrm{mg}$ Cr, Pall Mall = 0.93ng $/ \mathrm{mg}$ Cr, $\mathrm{p}=0.0427$ ) Finally, $\mathrm{Hg}$ levels differed between Marlboro brand and Newport brand smokers (Marlboro $=0.51 \mathrm{ng} / \mathrm{mg}$ Cr, Newport $=0.44 \mathrm{ng} / \mathrm{mg} \mathrm{Cr}, \mathrm{p}=0.0215)$. All statistically significant toxicant exposure differences are reported after adjustment for serum cotinine.

Table 2 shows regression weights for the individual level variables and cotinine in the model. Across markers, gender is a consistent predictor, generally higher in men than women. Blacks also differ from whites on most markers, most commonly having lower levels. Age appears to be related to NNAL and metal levels, but not all PAH metabolites. Cotinine is consistently and strongly positively associated with NNAL and PAH markers, but only shows a significant positive association with $\mathrm{Cd}$ and $\mathrm{Pb}$ among the metals. Given cotinine in the model, cigarettes per day is not consistently associated with biomarkers of exposure, excepting NNAL, where the highest level of consumption (>16 CPD) is associated with higher urine concentrations. Table 3 shows the model R-square values for the demographics-only model, demogrpahics plus 
CPD, demographics, CPD, and cotinine, and the full (including brand variables) models. The demographic factors in aggregate account for between 7 and 46 percent of variance in each marker. Adding CPD to the mdoel accounts for between 0 and 12 percent additional variance, and adding cotinine accounts for an additional 0 to 26 percent. This differs markedly across biomarkers - demographics, CPD, and cotinine account for $54 \%$ of variance in $\mathrm{HF}$, but only $10 \%$ of As.

Geometric means by brand family, tar group, and menthol status from the fully adjusted models (i.e., controlling for demographics, CPD, and cotinine) are shown in Table 4. Controlling for other factors, brand family was significantly associated with urinary levels of NNAL, 1HP, HFs, and HPHs. For NNAL, Marlboro and Pall Mall smokers had similar levels, and higher than smokers of the other brand families. For 1HP, HFs and HPHs the highest levels were seen in Pall Mall smokers, followed by Newport smokers. Tar group was associated with urinary $1 \mathrm{HP}, 2 \mathrm{HN}$ and $\mathrm{HPHs}$ concentrations - in all cases the relationship was inverse, such that smokers of brands yielding $6 \mathrm{mg}$ tar or less showed the highest concentrations. Menthol status was associated with urinary levels of NNAL with the highest levels among non-mentholated cigarettes. Adding brand information to the model accounted for an additional 2-8 percent of variance depending on the marker - effects were noticeably larger for PAH markers than for NNAL or the metals (see Table 3 ).

\section{DISCUSSION}

Our findings suggest few differences in exposure to nicotine, NNK, and PAHs among daily smokers of the leading US cigarette brands from 2007-2012, even after adjusting for menthol status, FTC tar group and person-level factors such as smoker's gender, age, race/ethnicity, and educational attainment. However, comparisons between models with and without brand information suggest that the primary drivers in exposure differences are individual level factors and cigarette consumption rather than product differences. That is, what you smoke appears to be far less determinative of exposure than how much you smoke.

Brand marketing does target different segments of the population, so it is not surprising that there are noted differences in the demographics of smokers of each brand. Younger smokers tended disproportionally to use Camel and Newport, while older smokers were more likely to use Marlboro and especially likely to use Pall Mall. Smokers of Marlboro, Camel and Pall Mall cigarettes were primarily NonHispanic White while those who smoked Newport cigarettes were usually Non-Hispanic Black. Pall Mall smokers were also more likely to smoke daily and to smoke 16 or more cigarettes per day. Thus, Pall Mall users tended to be heavier, more established smokers. These demographics are consistent with research on the use of premium and discount cigarette brands $^{34}$. Smokers of premium cigarette brands such as Marlboro and Newport have been found to be younger with a higher income, and smokers of discount cigarette brands such as Pall Mall were more likely to be older, middle and lower income, and heavier smokers ${ }^{34}$. Marlboro, Newport and Camel are the most heavily advertised cigarette brands and they are the preferred brands among adolescent and young adults, and mentholated cigarettes are marketed heavily towards the African American community ${ }^{21-26,39}$.

Other studies, including those using NHANES data, have looked at trends in serum cotinine concentrations ${ }^{40}$, as well as differences in serum cotinine levels seen among the major racial ethnic groups, age categories, gender and $\mathrm{BMI}^{6,41,42}$. NHANES data has also been used to look at differences in exposure levels for menthol versus non-menthol cigarettes ${ }^{43}$. Our findings showed similar trends for menthol versus nonmenthol cigarettes as Jones, et al., ${ }^{43}$ among the serum cotinine and urinary NNAL levels. In addition, Mendes, et al. ${ }^{44}$ looked at exposure levels among cigarette smokers according to their usual cigarette brand's smoking machine tar levels. Our study broadly replicates these prior studies and adds information on brand family-specific exposures. It is important to note specific-brand exposures, as many consumers are brand loyal, the tobacco industry's marketing tactics influence preferred brands ${ }^{21-26}$ and tobacco manufacturers are required to report HPHCs on a brand style-specific basis.

Per-cigarette yields and relative human exposure during smoking of different cigarette brands are not valid estimates of human exposure $\mathrm{e}^{45}$. It is known that nicotine needs vary from smoker to smoker and is related at least in part to the smoker's sociodemographic background $d^{4-6}$. In order to obtain desired nicotine levels, smokers will adjust their smoking habits to alter smoke intake accordingly ${ }^{46}$. For instance, smokers can take varying puff sizes and number of puffs per cigarette, and they can alter the ventilation of the cigarette by blocking filter perforations ${ }^{46}$. These compensatory smoking actions result in varying human smoke exposures ${ }^{46}$. Indeed, we saw in our analyses when brand tar levels were associated with biomarkers of exposure, the relationship was inverse, and smokers of lower tar brands had higher exposures. Our findings reiterate that individual brand emissions are poor estimators of smoker exposures. This is especially important to remember given recent reports that smokers of higher tar cigarettes showed more dependence symptoms ${ }^{47}$ - this may be an artifact of the 
sociodemographics of who self-selects to higher-tar products, rather than dependence producing features of those products.

We saw weak relationships between smoking or brand variables and indicators of metal exposure, other than $\mathrm{Cd}$, which is well established ${ }^{19,20}$. We saw a significant difference in Cd exposure by brand menthol status, but not brand family or tar yield, even controlling for other factors. Cotinine was not associated with smokers' urinary levels of As, Co, and Hg. Cigarettes are known to contain these metals, metal concentrations in tobacco can vary by brand/manufacturer, and they transfer into smoke. However, less is known about exposures directly attributable to smokers, and more work is clearly needed in this area.

Strengths of this study include the design of the NHANES program as it provides information collected from a nationallyrepresentative sample using standardized questionnaires that collects consistent content over time, including direct verification of brand smoked. However, there are some limitations. The questionnaire is subject to recall bias; the limited sample size does not provide the power to further examine the effects of specific demographic characteristics of current US smokers on smoke exposure; the cross-sectional time-series setup does not allow for assessment of individual change in exposure, or of brand switching. This may be particularly important for biomarkers with longer retention in the body. Additionally, urine PAH and metal data were available for only a subset of participants, meaning there is reduced power for detecting differences by brand. Finally, our models did not consider exogenous sources of PAH and metal exposures, such as environmental or occupational exposures. However, given the sample here are all cigarette smokers, the contributions of cigarettes are likely to be the most relevant point source, and adjusting for cigarettes smoked and cotinine level did account for substantial variance in most of these markers.

Our results suggest that the brand to brand differences seen in serum cotinine levels and other biomarkers indicative of cigarette smoking between the top US cigarette brands from 2007-2012 are primarily driven by individual differences among smokers, and to a lesser extent by differences among products.

\section{REFERENCES}

1. U.S. Department of Health and Human Services. The Health Consequences of Smoking-50 Years of Progress: A Report of the Surgeon General, 2014. Atlanta, GA: U.S. Department of Health and Human Services, Centers for Disease Control and Prevention, National Center for Chronic Disease Prevention and Health Promotion, Office on Smoking and Health; 2014. Available at: https://www.surgeongeneral.gov/library/reports/50-years-ofprogress/full-report.pdf (accessed February 2016)

2. U.S. Department of Health and Human Services. How Tobacco Smoke Causes Disease: The Biology and Behavioral Basis for Smoking-Attributable Disease: A Report of the Surgeon General. Atlanta, GA: U.S. Department of Health and Human Services, Centers for Disease Control and Prevention, National Center for Chronic Disease Prevention and Health Promotion, Office on Smoking and Health; 2010. Available at: http://whyquit.com/CDC/ SGR_2010_How_Tobacco_Smoke_Causes_Disease.pdf (accessed February 2016)

3. National Toxicology Program. Introduction to the Report on Carcinogens. Thirteenth Edition. U.S. Department of Health and Human Services, Public Health Service, National Toxicology Program; 2014:1-7.

4. National Cancer Institute. Risks Associated with Smoking Cigarettes with Low Machine Measured Yields of Tar and Nicotine. Smoking and Tobacco Control Monograph 13. Bethesda, MD: US Department of Health and Human Services, National Institutes of Health, National Cancer Institute; 2001. Available at: http://cancercontrol. cancer.gov/brp/TCRB/monographs/13/m13_complete.pdf. (accessed February 2016).

5. Fidler JA, Jarvis MJ, Mindell J, West R. Nicotine Intake in Cigarette Smokers in England: Distribution and Demographic Correlates. Cancer Epidemiol Biomarkers Prev. 2008;17(12): 3331-3336. doi: 10.1158/1055-9965.EPI-08-0296

6. Jarvis MJ, Giovino GA, O'Connor RJ, et al. Variation in nicotine intake among U.S. cigarette smokers during the past 25 years: evidence from NHANES surveys. Nicotine Tob Res. 2014;16(12):1620-1628. doi: $10.1093 / \mathrm{ntr} / \mathrm{ntu} 120$.

7. Centers for Disease Control and Prevention. Cotinine. National Biomonitoring Program. Available at: http://www.cdc.gov/ biomonitoring/Cotinine_BiomonitoringSummary.html. (accessed February 2016)

8. Kyerematen GA, Morgan ML, Chattopadhyay B, et al. Disposition of nicotine and eight metabolites in smokers and nonsmokers: identification of two metabolites that are longer lived than cotinine. Clin Pharmacol Ther 1990;48:641-651.

9. Jacob P, Yu L, Wilson M, Benowitz NL. Selected ion monitoring method for determination of nicotine, cotinine and deuteriumlabeled analogs: absence of an isotope effect in the clearance of (S)-nicotine-3',3'-d2 in humans. Biol Mass Spec. 1991;20:247-252.

10. Armitage AK, Dollery CT, George CF, et al. Absorption and metabolism of nicotine from cigarettes. Br Med J. 1975;4:313-316.

11. Hecht SS, Hoffmann D. Tobacco-specific nitrosamines, an important group of carcinogens in tobacco and tobacco smoke. Carcinogenesis. 1988;9(6):875-884.

12. Centers for Disease Control and Prevention. NNAL (4-(methylnitrosamino)-1-(3-pyridyl)-1-butanol). National Biomonitoring Program. Available at: http://www.cdc.gov/ biomonitoring/NNAL_FactSheet.html (accessed February 2016)

13. National Toxicology Program. N-Nitrosamines: 15 Listings. Report on Carcinogens. Thirteenth Edition. U.S. Department of Health and Human Services, Public Health Service, National Toxicology Program; 2014:1-23.

14. St. Helen G, Goniewicz ML, Dempsey D, et al. Exposure and Kinetics of Polycyclic Aromatic Hydrocarbons (PAHs) in Cigarette Smokers. Chem Res Toxicol. 2012;25(4):952-964. doi: $10.1021 / \mathrm{tx} 300043 \mathrm{k}$

15. Pappas RS, Fresquez MR, Watson CH. Cigarette smoke cadmium breakthrough from traditional filters: implications for exposure. J 
Anal Toxicol. 2015;39(1):45-51.

16. Pappas RS, Fresquez MR, Martone N, Watson CH. Toxic metal concentrations in mainstream smoke from cigarettes available in the USA. J Anal Toxicol. 2014;38(4):204-211.

doi: $10.1093 /$ jat/bku013.

17. Caruso RV, O'Connor RJ, Stephens WE, et al. Toxic metal concentrations in cigarettes obtained from U.S. smokers in 2009: results from the International Tobacco Control (ITC) United States survey cohort. Int J Environ Res Public Health. 2013;11(1):202217.

18. Afridi HI, Talpur FN, Kazi TG, Brabazon D. Effect of Trace and Toxic Elements of Different Brands of Cigarettes on the Essential Elemental Status of Irish Referent and Diabetic Mellitus Consumers. Biol Trace Elem Res. 2015;167(2):209-224.

19. Matsunaga Y, Agaku IT, Vardavas CI. The association between cigarette rod length, slim design, and blood cadmium levels among U.S. smokers: NHANES 1999-2010. Prev Med. 2014;65:87-91. doi: 10.1016/j.ypmed.2014.04.021.

20. Tellez-Plaza M, Navas-Acien A, Caldwell KL, et al. Reduction in cadmium exposure in the United States population, 1988-2008: the contribution of declining smoking rates. Environ Health Perspect. 2012;120(2):204-209.

21. Cummings KM, Hyland A, Bansal MA, et al. What do marlboro lights smokers know about low-tar cigarettes? Nicotine Tob Res. 2004;6(Suppl 3):S323-S332.

22. Kozlowski LT, Goldberg ME, Yost BA, et al. Smokers' misperceptions of light and ultra-light cigarettes may keep them smoking. Am J Prev Med. 1998;15(1):9-16.

23. Cummings KM, Hyland A, Pechacek TF, et al. Comparison of recent trends in adolescent and adult cigarette smoking behavior and brand preferences. Tob Control. 1997;6(Suppl 2):S31-S37.

24. Sharma A, Fix BV, Delnevo C, et al. Trends in market share of leading cigarette brands in the USA: national survey on drug use and health 2002-2013. BMJ Open. 2016;6(1):e008813. doi: 10.1136/bmjopen-2015-008813.

25. Centers for Disease Control and Prevention. Tobacco Brand Preferences. Smoking \& Tobacco Use. Available at: http://www.cdc. gov/tobacco/data_statistics/fact_sheets/tobacco_industry/brand_ preference/. (accessed February 2016)

26. Centers for Disease Control and Prevention. Cigarette Brand Preference Among Middle and High School Students Who Are Established Smokers --- United States, 2004 and 2006. MMWR. 2009:58(05);112-115.

27. U.S. Department of Health and Human Services, Food and Drug Administration, Center for Tobacco Products (CTP). Guidance for Industry and FDA Staff: "Harmful and Potentially Harmful Constituents" in Tobacco Products as Used in Section 904(e) of the Federal Food, Drug, and Cosmetic Act. 2011 (January): 1-5.

28. U.S. Department of Health and Human Services, Food and Drug Administration. Harmful and Potentially Harmful Constituents in Tobacco Products and Tobacco Smoke: Established List. 2012 (April): 20034-20037.

29. Centers for Disease Control and Prevention. National Health and Nutrition Examination Survey. About the National Health and Nutrition Examination Survey. Available at: http://www.cdc. gov/nchs/nhanes/about_nhanes.htm\#content. (accessed February 2016)

30. U.S. Department of Health and Human Services, Centers for Disease Control and Prevention, National Center for Health Statistics. National Health and Nutrition Examination Survey Biospecimen Program: NHANES III (1988-1994) and NHANES 1999-2014.
Vital and Health Statistics Series 2, Number 170. 2015 (July): 1-14. 31. U.S. Department of Health and Human Services, Centers for Disease Control and Prevention, National Center for Health Statistics. Key concepts About NHANES Survey Design. Available at: http://www. cdc.gov/nchs/tutorials/nhanes/SurveyDesign/SampleDesign/ Info1.htm. (accessed February 2016)

32. Centers for Disease Control and Prevention. National Health and Nutrition Examination Survey. NCHS Research Ethics Review Board (ERB) Approval. Available at: http://www.cdc.gov/nchs/ nhanes/irba98.htm. (accessed February 2016)

33. Centers for Disease Control and Prevention. National Health and Nutrition Examination Survey. Smoking - Cigarette Use. Available at: http://wwwn.cdc.gov/Nchs/Nhanes/2007-2008/SMQ_E.htm. (accessed February 2016)

34. Cornelius ME, Driezen P, Fong GT, et al. Trends in the Use of Premium and Discount Cigarette Brands: Findings from the ITC US Surveys (2002-2011). Tob Control. 2013;0:1-6.

35. Centers for Disease Control and Prevention. National Health and Nutrition Examination Survey. Blood and Urine Collection. Available at: http://www.cdc.gov/nchs/data/nhanes/nhanes_07_08/ labcomp_e.pdf. (accessed February 2016)

36. Centers for Disease Control and Prevention. National Health and Nutrition Examination Survey. Cotinine - Serum \& Total NNAL - Urine. Available at: http://wwwn.cdc.gov/Nchs/ Nhanes/2007-2008/COTNAL_E.htm. (accessed February 2016)

37. Centers for Disease Control and Prevention. National Health and Nutrition Examination Survey. Metals - Urine. Available at: http:// wwwn.cdc.gov/Nchs/Nhanes/2011-2012/UHM_G.htm. (accessed February 2016)

38. Centers for Disease Control and Prevention. National Health and Nutrition Examination Survey. Laboratory Procedures Manual. 2009 (July): 1-897.

39. Centers for Disease Control and Prevention. Smoking \& Tobacco Use. Tobacco Industry Marketing. Available at: http://www.cdc.gov/ tobacco/data_statistics/fact_sheets/tobacco_industry/marketing/. (accessed February 2016)

40. Jain RB. Trends in serum cotinine concentrations among daily cigarette smokers: data from NHANES 1999-2010. Sci Total Environ. 2014;472:72-77.

41. Caraballo RS, Giovino GA, Pechacek TF, et al. Racial and ethnic differences in serum cotinine levels of cigarette smokers: Third National Health and Nutrition Examination Survey, 1988-1991. JAMA. 1998;280(2):135-139.

42. Roethig HJ, Munjal S, Feng S, et al. Population estimates for biomarkers of exposure to cigarette smoke in adult U.S. cigarette smokers. Nicotine Tob Res. 2009;11(10):1216-1225.

43. Jones MR, Apelberg BJ, Tellez-Plaza M, et al. Menthol cigarettes, race/ethnicity, and biomarkers of tobacco use in U.S. adults: the 1999-2010 National Health and Nutrition Examination Survey (NHANES). Cancer Epidemiol Biomarkers Prev. 2013;22(2):224232.

44. Mendes P, Liang Q, Frost-Pineda K, et al. The relationship between smoking machine derived tar yields and biomarkers of exposure in adult cigarette smokers in the US. Regul Toxicol Pharmacol. 2009;55(1):17-27.

45. World Health Organization. The Scientific Basis of Tobacco Product Regulation: Second Report of a WHO Study Group. WHO Press; 2008: 1-289..

46. Kozlowski LT, O'Connor RJ. Cigarette filter ventilation is a defective design because of misleading taste, bigger puffs, and blocked vents. Tob Control. 2002;11(Suppl 1):i40-i50. 


\section{Research Paper}

47. Redner R, White TJ, Bunn JY, Higgins ST Use of High-Nicotine/ Tar-Yield (Full-Flavor) Cigarettes and Risk for Nicotine Dependence in Nationally Representative Samples of US Smokers. Nicotine Tob Res. 2016 Jun;18(6):1424-30.

CONFLICT OF INTERESTS All the authors have completed and submitted the ICMJE Form for Disclosure of Potential Conflicts of Interest and none were reported.

FUNDING

There was no source of funding for this research.

PROVENANCE AND PEER REVIEW

Not commissioned;

Externally peer reviewed 